\title{
EFECTOS AMBIENTALES DEL REEMPLAZO DE BOSQUE NATIVO POR PLANTACIONES (ESTUDIO EN CUATRO MICROCUENCAS EN LA PROVINCIA DE VALDIVIA)
}

\author{
Luis Otero D. $(*)$ \\ Alvaro Contreras J. ("*) \\ Luis Barrales M. (*t)
}

RESUMEN

En cuatro cuencas vecinas ubicadas en el Valle Central de la Provincia de Valdivia, se estudió el efecto de la sustitución de bosque Nativo por plantaciones de Pinus radiata sobre la calidad y producción de agua. Para ello se recogió información cuantitativa de precipitación, descarga y producción de sedimentos durante 17 meses. La selección de estas cuencas se realizó en base al método de las cuencas pares, con condiciones de similitud en cuanto a tamaño, geomorfologia y clima.

El rendimiento hídrico promedio de las cuencas cubiertas por vegetación nativa fue hasta un $28 \%$ superior que el rendimiento de las cuencas con pino, para las estaciones del año con bajas precipitaciones. Las cuencas con bosque nativo entregaron caudales inferiores que las cuencas con pino en los periodos de máxima precipitación, 274 $\mathrm{Ls} / \mathrm{Km}^{2}$ y $359 \mathrm{Ls} / \mathrm{Km}^{2}$, respectivamente.

La concentración de sedimentos en suspensión entregó niveles superiores en las cuencas cubiertas por pino, alcanzando a $7.5 \mathrm{mg} / \mathrm{L}$ en el análisis de turbidez y 76,13 $\mathrm{mg} / \mathrm{L}$ en sedimentos totales. Las cuencas cubiertas por vegetación nativa alcanzaron niveles máximos de 6,0 y $41,41 \mathrm{mg} / \mathrm{L}$ en turbidez y sedimentación, respectivamente.

Se concluye que existe una tendencia de regulación de los caudales en las cuencas cubiertas con bosque nativo en comparación con las cubiertas por plantaciones de pino, entregando caudales superiores en las estaciones del año con déficit de lluvia y menores en los periodos de intensas precipitaciones. Este efecto regulador también se ve reflejado en la concentración de sedimentos en suspensión.

(*) Ingeniero Forestal Mg. Instituto Forestal Sede Valdivia.

(*) Ingeniero Forestal. Instituto Forestal Sede Valdivia.

(**) Técnico Forestal. Instituto Forestal Sede Valdivia. 


\section{ABSTRACT}

In four basins nearby, placed in the Central Valley of the province of Valdivia, it was studied the effect of the substitution of the native forest for plantations of radiata pine about the quality and production of water. For this, was obtained a quantitative compilation of information about rainfall, discharge and production of sediments during 17 months. The selection of these basins, with similar conditions in size, geomorphology and climate.

The average hydric yield of the basins covered by native vegetation was up to $28 \%$ higher than the yield of the basins with radiata pine, in low rainfall seasons. The basins with native forest gave inferior flows than the basins with radiata pine in the high rainfall season, $274 \mathrm{Ls} / \mathrm{Km}^{2}$ and $359 \mathrm{~L} / \mathrm{Km}^{2}$, respectively.

The concentration of the sediments in suspension gave higher levels in the basins covered by pine, reaching up to $7,5 \mathrm{mg} / \mathrm{L}$ in the analysis of mud and $76,13 \mathrm{mg} / \mathrm{L}$ in total sediments. The basins covered by native vegetation reached maximum level of $6,0 \mathrm{y}$ $41,41 \mathrm{mg} / \mathrm{L}$ in mud and sedimentation respectively.

It is concluded that there is a tendency to control the flows in the basins covered with native forests in comparison with the ones covered with plantations of pine, thus giving flows higher in the seasons with deficit of rain, and lower in period of intensive rainfall. This regulating effect is also reflected in the concentration of sediments in suspension. 


\section{INTRODUCCION}

La vegetación posee una gran influencia sobre los montos de precipitación que llegan al suelo, su distribución y sobre el agua que alcanza a los cauces, por lo que cualquier transformación de magnitud que se realice en la vegetación se manifestará en los montos de agua que producen las cuencas y en su calidad (Lassen et al 1965; Lopez y Blanco 1976; Lopez y Pérez 1981; Huber et al 1985; Huber 1992).

En cuencas destinadas a uso forestal maderero muchas veces se debe compatibilizar el aprovechamiento de este recurso con la obtención de otros bienes, como recreación y producción de agua, por lo cual se debe considerar que las alteraciones que se produzcan en el suelo o vegetación presente en las cuencas tendrá repercusiones en sus recursos hídricos.

Los efectos producidos por el manejo de la vegetación en los recursos hídricos de la Región han sido tema de estudio (Iroume 1990; Rodríguez 1991; Iroume et al 1992), como también la tasa de consumo hídrico de algunas cubiertas forestales (Huber et al 1985; Huber 1992). Sin embargo, no existen estudios de los efectos producidos por el reemplazo de bosque nativo por plantaciones de pino, sobre la cantidad y calidad del agua que llega a sus cauces.

El objetivo de este estudio es determinar el efecto de la sustitución de bosque nativo por plantaciones de pino en el rendimiento y calidad del agua que producen las cuencas. 


\section{METODOLOGIA}

\section{Area de Estudio.}

El área de estudio corresponde a cuatro cuencas forestales vecinas, dos de ellas cubiertas con vegetación nativa y las otras dos por plantaciones de pino. Las cuencas se encuentran ubicadas 5 kilómetros al sur de la ciudad de Los Lagos en la Provincia de Valdivia, $39^{\circ} 55^{\prime}$ LS y $72^{\circ} 47^{\circ}$ LW (Figuras $N^{\circ} \mathrm{S} 1$ y 2 ).

La altitud media de las cuencas es de $150 \mathrm{msnm}$, y sus pendientes medias oscilan entre los $31 \%$ y $36 \%$, con orientación general noroeste y suroeste.

El sustrato geológico corresponde a la formación Piedra Laja, propia de la Cordillera de la Costa y de la parte central de la Provincia de Valdivia, compuesta de rocas donde predominan los esquistos micáceos. Sobre este material base se ha depositado una capa continua de ceniza volcánica de espesor variable.

Los suelos de este sector se consideran Trumaos jóvenes y, aparentemente, de dos series de suelos, Malihue y Correltue, ambas de gran parecido por sus características físicas y quimicas.

Según INIA (1989), el sector de Lipingue se ubica en la zona agroclimática denominada Purranque, esta zona se caracteriza por una temperatura media anual de $10,9^{\circ} \mathrm{C}$, con una máxima media del mes más cálido (enero) de 21,4 ${ }^{\circ} \mathrm{C}$ y una mínima media del mes más frió (junio) de $3,0^{\circ} \mathrm{C}$. La precipitación alcanza una media anual de $1542 \mathrm{~mm}$ y el mes más lluvioso corresponde a mayo, con $276 \mathrm{~mm}$. No se presenta estación seca.

La superficie de las cuencas Pino 1 y Pino 2 es de 0,99 y $0,75 \mathrm{~km}^{2}$ y la Nativo 1 y Nativo 2 es de 0,54 y $1,32 \mathrm{~km}^{2}$ respectivamente.

Las cuencas con pino están cubiertas mayoritariamente por plantaciones de 8 a 16 años de edad, con pequeños sectores alrededor de las quebradas con vegetación nativa. Las cuencas con bosque nativo poseen renovales de roble, laurel y bosques adultos remanentes de olivillo y ulmo mezclados con matorral (Otero et al, 1993). 


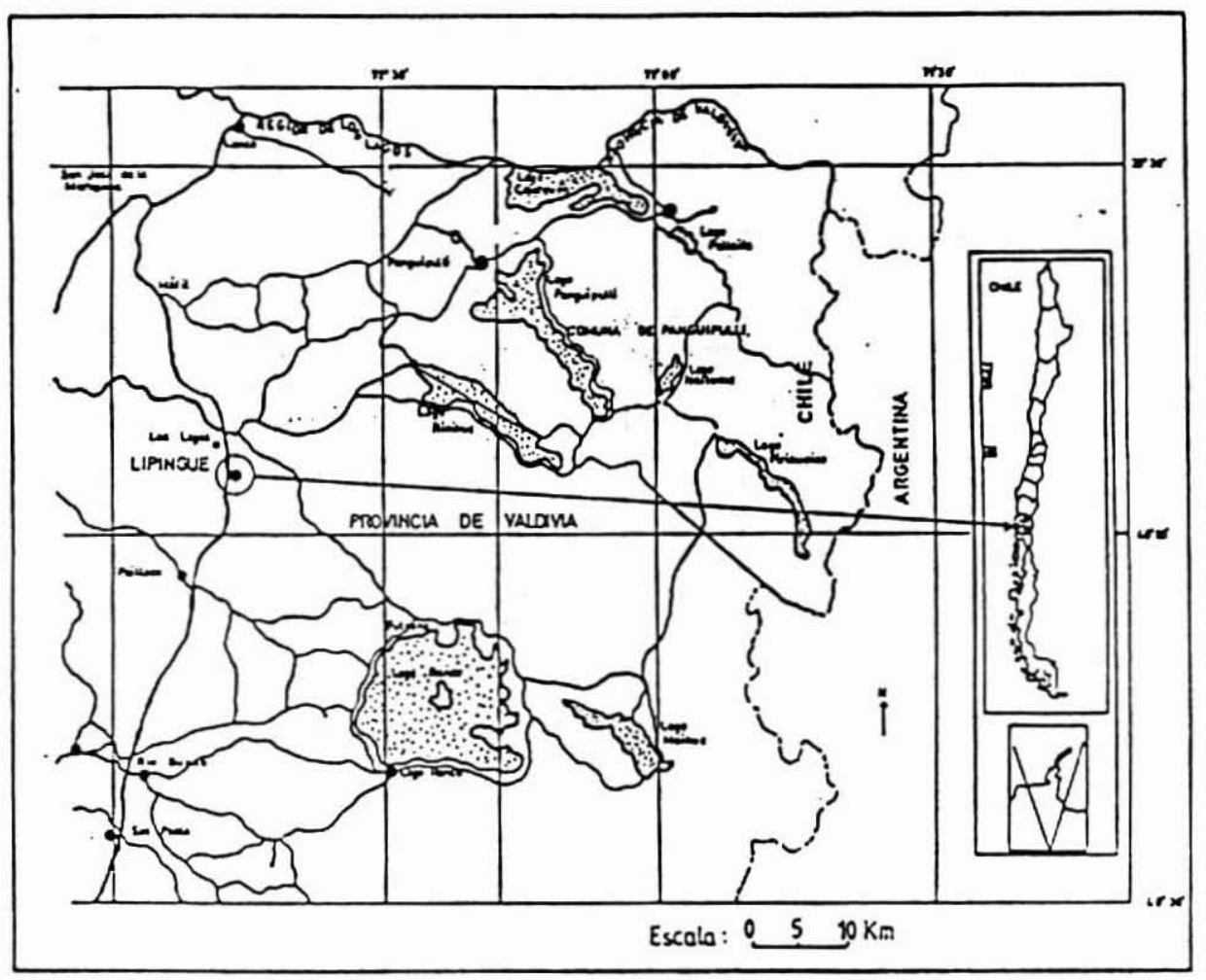

Figura N ${ }^{\circ}$ 1. UBICACION DEL AREA DE ESTUdIO 


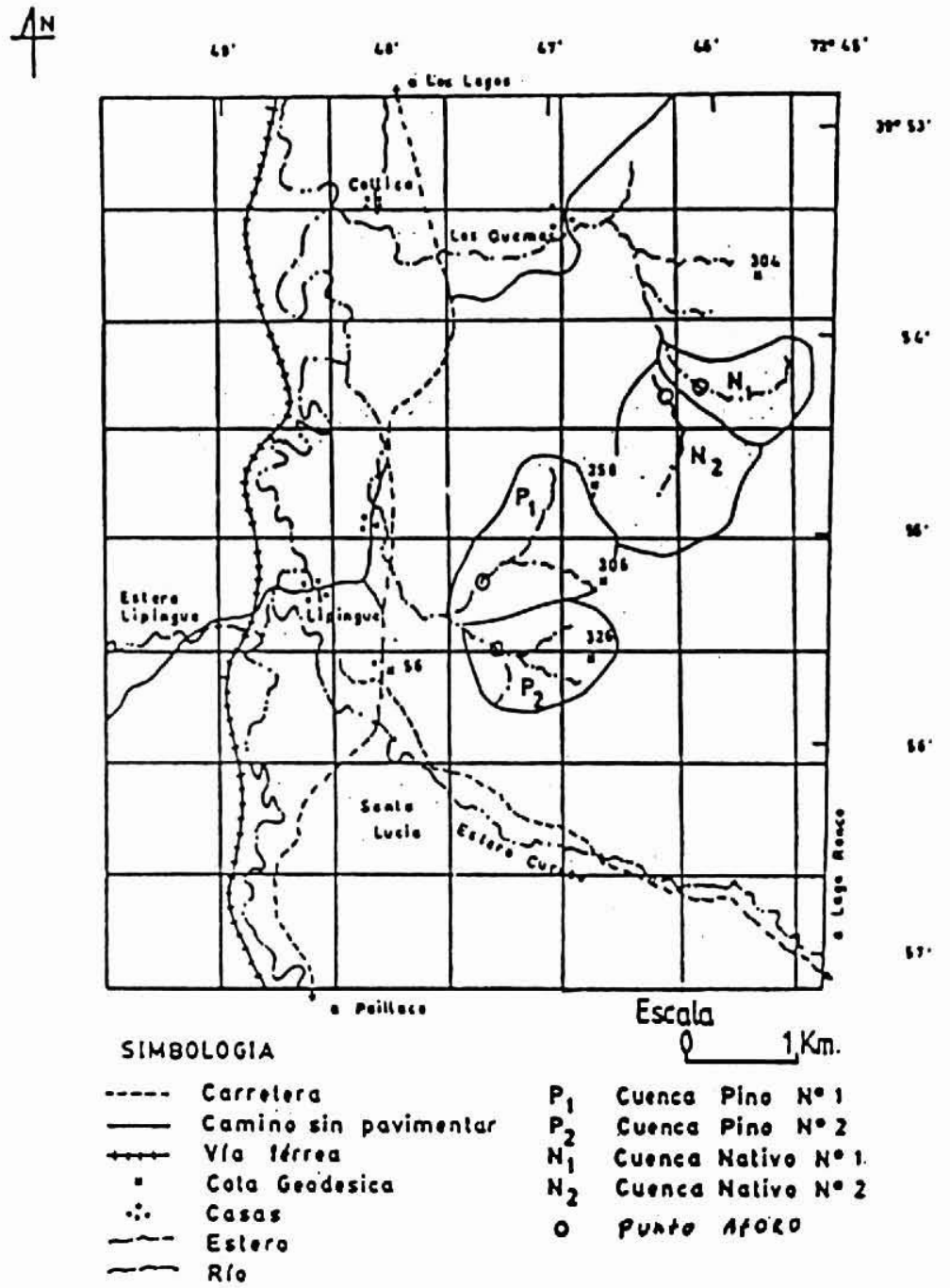

Figura No 2. UBICACION CUENCAS DE LIPINGUE 
Cuenca Nativo 2 : 34 análisis de turbidez en (mg/L) 13 de seston en $(\mathrm{mg} / \mathrm{L})$

Variables y Análisis de Laboratorio.

Nivel de los Cauces y Caudal.

La determinación de caudales se realizó mediante el método de "Aforo con Flotador" propuesto en diferentes estudios (Contreras, en prensa; Ciancaglini, 1987; FAO, 1976). Bajo este método el caudal está dado por la siguiente expresión:

$Q \quad=V * A$

$Q=$ Caudal $\left(\mathrm{m}^{3} / \mathrm{h}\right)$

$\mathrm{V}=$ Velocidad media de escurrimiento $(\mathrm{m} / \mathrm{s})$

$A=$ Sección o área de escurrimiento $\left(\mathrm{m}^{2}\right)$

$V=V \cdot S * 0,85$

V. $S=$ Velocidad media superficial

El área o sección de escurrimiento se obtuvo en cada aforo, para lo cual se midió la altura de la corriente de agua en diferentes puntos en su sección transversal.

La velocidad media superficial se obtuvo midiendo una distancia en la corriente en sentido longitudinal $(3-6 \mathrm{~m})$ en el punto de aforo, en este transecto se lanzo un flotador y se tomo el tiempo que transcurrió en recorrer la distancia. Se realizó esta operación 5 veces y se obtuvo una media. La velocidad media superficial se multiplicó por 0,85 (Coeficiente de rugosidad de la sección de escurrimiento), con lo cual se obtuvo la velocidad media de escurrimiento.

Para facilitar las mediciones y disminuir el error, se regularizaron las secciones de escurrimiento para cada vertiente en la sección de aforo, 


\section{Toma de Datos.}

Este estudio se desarrolló en el período comprendido entre el 8 de enero de 1993 y 31 de mayo de 1994.

Durante el periodo de medición se registraron precipitaciones diarias utilizando un pluviómetro instalado a campo abierto, a $200 \mathrm{~m}$ del área de estudio, en un predio particular colindante a las cuencas.

En las secciones de aforo se llevó un registro periódico de niveles cada dos o tres semanas, el número de registros para las diferentes cuencas es el siguiente.

\section{Cuenca Pino $1: 36$ registros \\ Cuenca Pino 2 : 36 registros \\ Cuenca Nativo $1: 37$ registros \\ Cuenca Nativo $2: 37$ registros}

En las secciones de aforo se tomaron muestras de agua de un litro, para determinar turbidez y sedimentos totales (seston total). Variables comúnmente medidas para determinar calidad del agua en estudios de manejo de cuencas (Mac Donald, 1991). Las muestras se obtuvieron del centro del cauce a una profundidad intermedia, en dos puntos, de tal forma de evitar la posible sedimentación de los bordes y del fondo del cauce.

Los análisis de turbidez efectuados corresponden casi en su totalidad al número de registros de niveles y el de sedimentación a los eventos de importancia en el período estudiado.

Cuenca Pino 1 : 34 análisis de turbidez en $(\mathrm{mg} / \mathrm{L}) 15$ de seston en $(\mathrm{mg} / \mathrm{L})$

Cuenca Pino 2 : 35 análisis de turbidez en ( $\mathrm{mg} / \mathrm{L}) 14$ de seston en (mg/L)

Cuenca Nativo 1 : 35 análisis de turbidez en $(\mathrm{mg} / \mathrm{L}) 14$ de seston en $(\mathrm{mg} / \mathrm{L})$ 
mediante la instalación de una estructura de madera y cemento de aproximadamente $2 \mathrm{~m}$ de largo y entre 40 a $70 \mathrm{~cm}$ de altura de acuerdo a la profundidad del lecho de cada cuenca, de tal forma que resistiera las crecidas sin socavar las orillas. También se instaló una vara graduada al centro del cauce con el objeto de medir niveles, en forma rápida.

Turbidez y Sedimentación.

La determinación de turbidez se realizó a través de un espectrógrafo, y los resultados se entregaron en miligramos por litro. Para la obtención de valores de sedimentos totales se hicieron estudios de sedimentación con la técnica de seston (Mac Donald, 1991). El análisis de las muestras se realizó en el Instituto de Zoologia de la Universidad Austral.

Análisis Estadistico.

Con el objeto de validar estadísticamente las diferencias entre las cuencas se realizaron análisis de varianza para las variables caudal, turbidez y seston.

Para las variables turbidez y seston no se consideraron todos los valores, especialmente los que representan niveles más bajos de caudal y los valores 0 puntos fuera de rango de los datos. Ello se realizó con el objeto de normalizar la distribución de los datos y debido a que al hacer el análisis de turbidez el minimo valor que se puede obtener es $2,5 \mathrm{mg} / \mathrm{L}$, por esta razón un alto número de análisis entregaron esta cantidad, la cual se registró principalmente para los niveles más bajos de caudal.

En cuanto a la variable caudal estos datos se estratificaron en dos grupos, menores de $60 \mathrm{~L} / \mathrm{s} / \mathrm{Km}^{2}$ y superiores a este nivel. Se utilizó esta metodología debido a que la gran diferencia entre caudales de invierno y verano no permite determinar grupos comparables para un análisis a través de las medias.

Para estudiar en mejor forma la distribución de los datos de los caudales se realizó un análisis de frecuencia para los caudales promedio de las cuencas de pino y nativo. 


\section{RESULTADOS}

Análisis de la Precipitación.

La precipitación para el año 1993 fue de $2705 \mathrm{~mm}$ y para el período de enero a mayo de 1994 fue de $584 \mathrm{~mm}$, sumando en total $3289 \mathrm{~mm}$. La precipitación caida de enero a mayo de 1994 fue inferior en un $50 \%$ a la registrada en el año 1993 a igual fecha (Figura №3).

La distribución de la precipitación diaria se presenta en la Figura №4, donde se aprecian los eventos de importancia y los dias sin precipitación a lo largo del periodo de estudio.

Para el año 1993 las precipitaciones se mantuvieron bajas en los meses de enero a febrero, produciéndose un aumento paulatino en la segunda semana del mes de marzo, llegando a los dias 16 y 17 con un evento de importancia de 93 y $31 \mathrm{~mm}$, respectivamente.

Entre los meses de abril a julio se produjeron las máximas precipitaciones mensuales con un total de $1800 \mathrm{~mm}$, que representan el $66,54 \%$ de la precipitación del año 1993. El mes de abril presentó entre los días 11 al 12 un evento de importancia con 77 y $94 \mathrm{~mm}$, respectivamente.

Mayo no presentó precipitaciones diarias de alta intensidad, pero llovió el $80 \%$ de los dias del mes, con un total de $469 \mathrm{~mm}$, por lo cual este mes fue uno de los más lluviosos dentro del período de estudio.

El mes de junio fue el de mayor precipitación del año 1993 con $524 \mathrm{~mm}$ (Figura $\mathrm{N}^{\circ} 3$ ), concentrándose $367 \mathrm{~mm}$ entre los dias 19 al 30 de este mes y encontrándose el dia 14 como el de máxima precipitación anual con $140 \mathrm{~mm}$.

Julio fue también un mes de alta precipitación, cayeron $157 \mathrm{~mm}$ entre los dias 1 al 9, presentando dos eventos de importancia; del 16 al 18 con $205 \mathrm{~mm}$ y el dia 25 con $70 \mathrm{~mm}$.

A partir de agosto comienza el decrecimiento de las precipitaciones, propio de la primavera, presentándose solo un evento de importancia en los días $\mathbf{3 0}$ de noviembre y 01 de diciembre, con 78 y $29 \mathrm{~mm}$, respectivamente, disminuyendo en el transcurso del mes de diciembre. 
Enero a marzo de 1994 fue un período bastante seco, con bajas precipitaciones. En abril y mayo se produjeron dos eventos de importancia, uno se registro el dia 29 de abril con $64 \mathrm{~mm}$ y el otro ocurrió el dia 23 de mayo con $64 \mathrm{~mm}$.

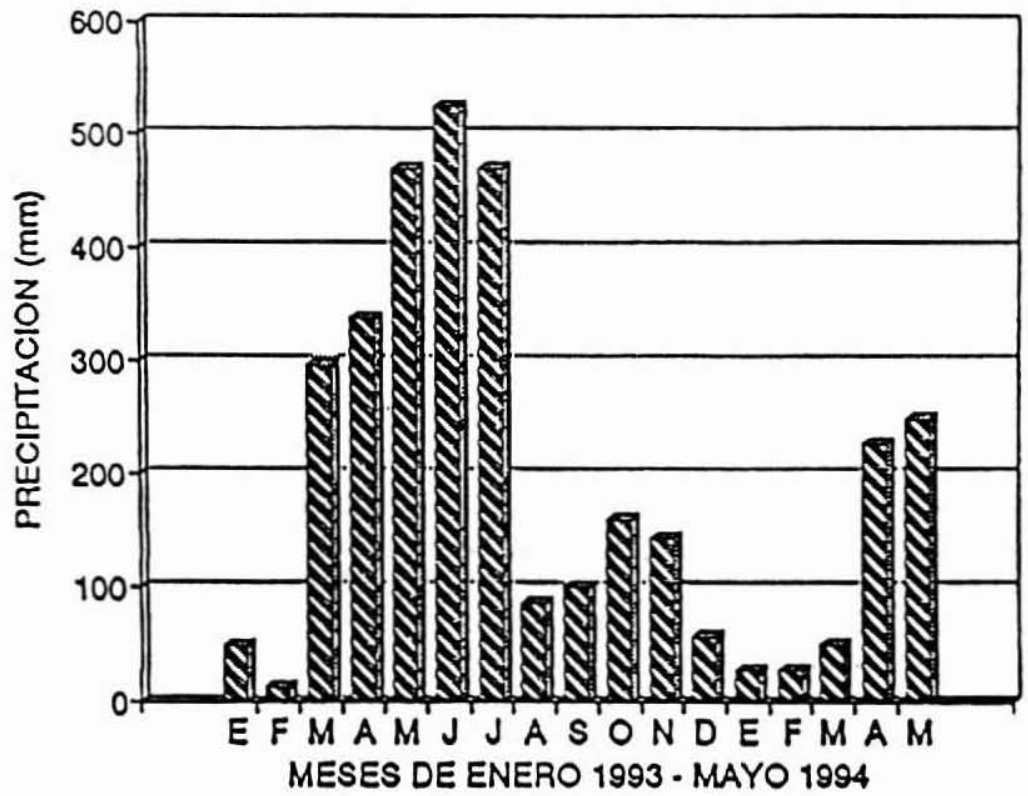

Figura $N^{\circ} 3$. DISTRIBUCION DE LA PRECIPITACION MENSUAL

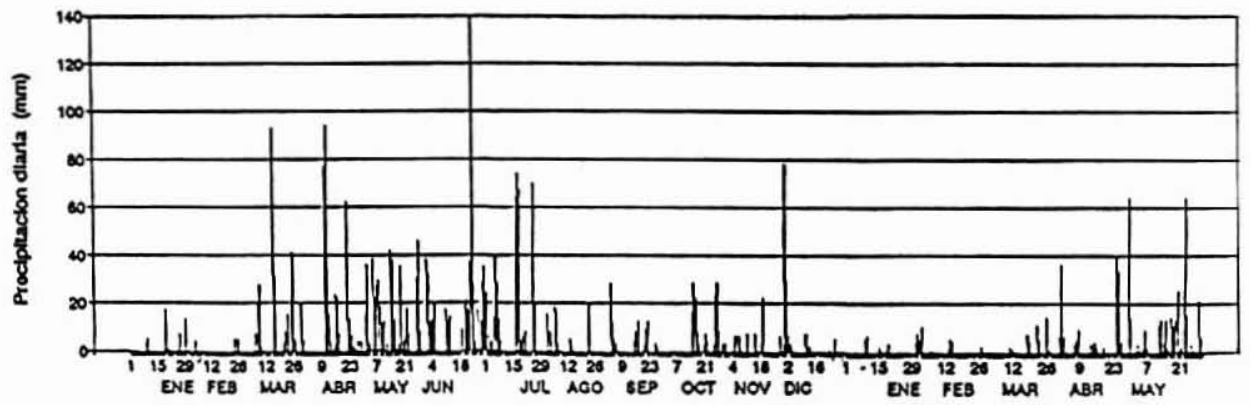

Figura N ${ }^{0} 4$. DISTRIBUCION DE LA PRECIPITACION DIARIA 


\section{Rendimiento Hidrico.}

Caudales por Cuenca.

La estimación de caudales especificos en litros por segundo por kilómetro cuadrado de las cuencas $\left(\mathrm{L} / \mathrm{s} / \mathrm{km}^{2}\right)$ se presentan en el Cuadro $\mathrm{N}^{0} 1$.

En la Figura $\mathrm{N}^{\circ} 5$, se aprecia la distribución de los caudales especificos para las cuatro cuencas, durante el período de estudio.

- Verano.

El comportamiento de los caudales en las cuencas pino y nativo muestra un decrecimiento desde el inicio del periodo de estudio hasta el dia 53 (2 de marzo), llegando a niveles minimos de 27,26 y $17,68 \mathrm{~J} / \mathrm{km}^{2}$, en las cuencas de pino, y de 17,34 y $25,46 \mathrm{~L} / \mathrm{s} / \mathrm{km}^{2}$, en las cubiertas por bosque nativo. Estos valores son semejantes a los encontrados en cuencas cercanas a Valdivia (Contreras, en prensa) que entregaron niveles mínimos de $17,51 \mathrm{Ls} / \mathrm{km}^{2}$, en una cuenca cubierta principalmente por plantaciones de pino, y entre 12,5 y $22,11 \mathrm{Ls} / \mathrm{km}^{2}$ para cuencas cubiertas principalmente por bosque nativo. Otro estudio realizado en la zona registró un valor mínimo de caudal de 13,36 $\mathrm{L} / \mathrm{km}^{2}$ el dia 6 de marzo 1990 (Rodríguez, 1991). La disminución de los niveles en esta época del año se debe a las bajas precipitaciones, que sólo coparian algunas fuentes de déficit (intercepción, recarga de humedad del suelo), y no tendrian la capacidad de generar escorrentias superficiales de importancia.

- Otoño.

El primer aumento brusco en los caudales en las cuatro cuencas monitoreadas fue el dia 68 (17 de marzo), desde niveles de $30 \mathrm{~L} / \mathrm{km}^{2}$ hasta 154 y $220 \mathrm{~L} / \mathrm{s} / \mathrm{km}^{2}$ en las Pino 2 y Pino 1 , respectivamente y entre 118 y 151 
$\mathrm{U} / \mathrm{km}^{2}$ en las cuencas Nativo 2 y Nativo 1, respectivamente. Este aumento se generó debido a las altas precipitaciones del primer evento de importancia del año que entregó un total de $124 \mathrm{~mm}$ en 48 horas.

- Invierno.

Un segundo evento de importancia se presentó el dia 132 (20 de mayo) con caudales específicos de 183 y $359 \mathrm{~L} / \mathrm{s} / \mathrm{km}^{2}$ para las cuencas de Pino 2 y Pino 1 , respectivamente, y entre 205 y $274 \mathrm{~L} / \mathrm{s} / \mathrm{km}^{2}$ para las cuencas Nativo 1 y Nativo 2 , respectivamente. Estos elevados valores de caudal se deben a que previo al evento hubo tres semanas con precipitaciones en casi la totalidad de los días, lo cual ha debido copar la capacidad de almacenamiento de agua en el suelo, generando escorrentias de importancia, siendo las más elevadas dentro del periodo de estudio.

Un tercer evento, aunque no con caudales específicos de gran magnitud, ocurrío el día 228 (23 de agosto) de $19 \mathrm{~mm}$, después de dos semanas con escasas precipitaciones.

- Primavera.

El cuarto evento de importancia medido en los caudales especificos ocurrió el día 327 ( 01 de diciembre), en el cual los caudales alcanzaron niveles de 178 y $270 \mathrm{~L} / \mathrm{sm}^{2}$ en las cuencas Pino 2 y Pino 1 , respectivamente, y entre 165 y $302 \mathrm{~L} / \mathrm{s} / \mathrm{km}^{2}$ en las cuencas Nativo 1 y Nativo 2, respectivamente. Este brusco aumento se debió a las intensas precipitaciones caídas en los días 30 de noviembre y 01 de diciembre, que en total alcanzaron a los $107 \mathrm{~mm}$.

Del análisis de la Figura $N^{0} 5$ se desprende que los máximos caudales registrados se produjeron en la cuenca de Pino 1 la que alcanzó el mayor caudal específico el dia 132 (20 de mayo) con $359 \mathrm{~L} / \mathrm{s}^{\mathrm{km}} \mathrm{km}^{2}$. En las cuencas de Nativo el mayor caudal registrado ocurrió el día 327 ( 01 de Diciembre) en el cual la cuenca de Nativo 1 alcanzo un caudal de $302 \mathrm{~L} / \mathrm{s} / \mathrm{km}^{2}$. 


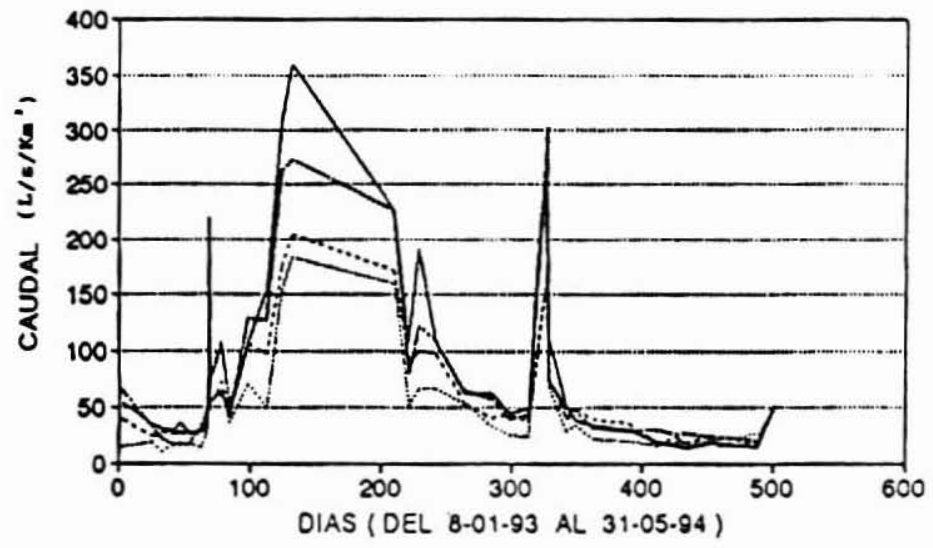

- PINO 1 -. PINO 2 NATIVO 1 ...... NATIVO 2

Figura $N^{\circ}$ 5. DISTRIBUCION DE LOS CAUDALES ESPECIFICOS 


\section{Cuadro $\mathrm{N}^{0} 1$}

CAUDALES ESPECIFICOS EN LAS DIFERENTES CUENCAS (U/s/km²)

\begin{tabular}{|c|c|c|c|c|c|c|c|}
\hline Dia/Mes & $N^{0}$ Dias & Pino 1 & Pino 2 & Nativo 1 & Nativo 2 & $\begin{array}{c}\text { Pino } \\
\text { Promedio }\end{array}$ & $\begin{array}{c}\text { Nativo } \\
\text { Promedio }\end{array}$ \\
\hline $08-01$ & 1 & & & 62,58 & 40.18 & & 51.38 \\
\hline $11-01$ & 3 & 54,03 & 15,40 & 64,87 & 40,18 & 34,72 & 52,53 \\
\hline $02-02$ & 25 & 35,09 & 19,18 & 33,28 & 24,01 & 27,14 & 28,65 \\
\hline $10-02$ & 33 & 31,50 & 10,09 & 22,52 & 29,05 & 20,80 & 25,79 \\
\hline $18-02$ & 41 & 29,82 & 17,68 & 17,34 & 25,46 & 23,75 & 21,40 \\
\hline $23-02$ & 46 & 36,24 & 19,18 & 17,34 & 27,24 & 27,71 & 22,29 \\
\hline $02-03$ & 53 & 27.26 & 17,68 & 17.34 & 25,46 & 22,47 & 21,40 \\
\hline $12-03$ & 63 & 30,98 & 15,02 & 34,76 & 28,99 & 23,00 & 31,88 \\
\hline $16-03$ & 67 & 42,07 & 30,19 & 51,23 & 31,96 & 36,13 & 41,60 \\
\hline $17-03$ & 68 & 220,67 & 154,16 & 151,78 & 118,09 & 187,42 & 134,94 \\
\hline $18-03$ & 69 & 54,75 & 53,31 & 72,06 & 46,37 & 54,03 & 59,22 \\
\hline $26-03$ & 77 & 65,04 & 61,66 & 107,05 & 75,03 & 63,35 & 91,04 \\
\hline $02-04$ & 84 & 42,52 & 35,12 & 49,08 & 42,52 & 38,82 & 45,80 \\
\hline $15-04$ & 97 & 105,88 & 70,75 & 128,54 & 106,65 & 88,32 & 117,60 \\
\hline $30-04$ & 112 & 153,52 & 49,58 & 126,86 & 96,01 & 101,55 & 111,44 \\
\hline $12-05$ & 124 & 307,33 & 153,25 & 263,70 & 174,67 & 230,29 & 219,19 \\
\hline $20-05$ & 132 & 359,85 & 183,18 & 274,02 & 205,26 & 271,52 & 239,64 \\
\hline $06-08$ & 210 & 226,73 & 158,75 & 225,89 & 171,56 & 192,74 & 198,73 \\
\hline $17-08$ & 221 & 107,59 & 52,11 & 81,54 & 88,22 & 79,85 & 84,88 \\
\hline $24-08$ & 228 & 191,56 & 67,03 & 121,35 & 101,20 & 129,30 & 111,28 \\
\hline $07-09$ & 242 & 109,87 & 67,03 & 108,10 & 98,54 & 88,45 & 103,32 \\
\hline $29-09$ & 264 & 61,67 & 53,38 & 64,99 & 53,89 & 57,53 & 59,44 \\
\hline $19-10$ & 284 & 63,70 & 33,15 & 58,21 & 40,72 & 48,43 & 49,47 \\
\hline 03-11 & 299 & 44,59 & 25,14 & 40,07 & 43,43 & 34,87 & 41,75 \\
\hline $17-11$ & 313 & 48,79 & 24,29 & 43,24 & 36,80 & 36,54 & 40,02 \\
\hline $01-12$ & 327 & 270,92 & 178,73 & 302,40 & 165,23 & 224,83 & 233,82 \\
\hline $02-12$ & 328 & 114,25 & 67,91 & 73,69 & 79,80 & 91,08 & 76,75 \\
\hline $16-12$ & 342 & 51,81 & 28,32 & 50,20 & 39,66 & 40,07 & 44,93 \\
\hline $22-12$ & 348 & 37,79 & 34,11 & 47,82 & 49,22 & 35,95 & 48,52 \\
\hline $05-01$ & 362 & 34,24 & 22,12 & 32,29 & 39,81 & 28,18 & 36,05 \\
\hline $02-02$ & 390 & 30,89 & 20,22 & 29,40 & 36,59 & 25,56 & 33,00 \\
\hline $24-02$ & 412 & 18,91 & 16,77 & 30,41 & 19,24 & 17,84 & 24,83 \\
\hline $11-03$ & 427 & 16,74 & 18,99 & 26,46 & 22,84 & 17,87 & 24,65 \\
\hline 17.03 & 433 & 14,41 & 19,55 & 27,84 & 18,72 & 16,98 & 23,28 \\
\hline $04-04$ & 451 & 18.07 & 20,31 & 23,94 & 24,75 & 19,19 & 24,35 \\
\hline 12.05 & 489 & 15.14 & 27,48 & 20,77 & 18,20 & 21,31 & 19,49 \\
\hline 31.05 & 501 & 53,36 & 47,94 & 49,63 & 51,96 & 50,65 & 50,80 \\
\hline
\end{tabular}


Caudales Promedio, Nativo,

En la Figura $N^{\circ} 6$ se muestran los caudales promedios de las cuencas Pino y

Durante la época de menor precipitación los caudales promedios de las cuencas Nativo son superiores a las Pino, hasta el dia en que se produce el primer evento de importancia en las precipitaciones, donde los caudales máximos ocurren en las cuencas Pino, Una vez que disminuyen las precipitaciones los caudales de Nativo se hacen superiores hasta que se produce otro evento de importancia en las lluvias, donde las cuencas cubiertas por pino vuelven a entregar el máximo caudal,

Este comportamiento podria deberse a que los bosques con vegetación nativa poseen la facultad de regular en mejor forma los niveles de escorrentía cuando las precipitaciones son de alta intensidad, ya sea por una mayor capacidad de interceptar las precipitaciones o bien por su mejor capacidad de infiltración y almacenamiento (Huber, 1992), Este aumento de las reservas de agua en el suelo en los bosques nativos y las mayores tasas de evapotranspiración de los bosques de pino (Alvarez, 1986; Huber, 1992; Francke, 1993) se ve reflejado en un mayor rendimiento hídrico en las cuencas Nativo en las estaciones del año con bajas precipitaciones, alcanzando niveles hasta un $28 \%$ superiores que las cuencas Pino, 


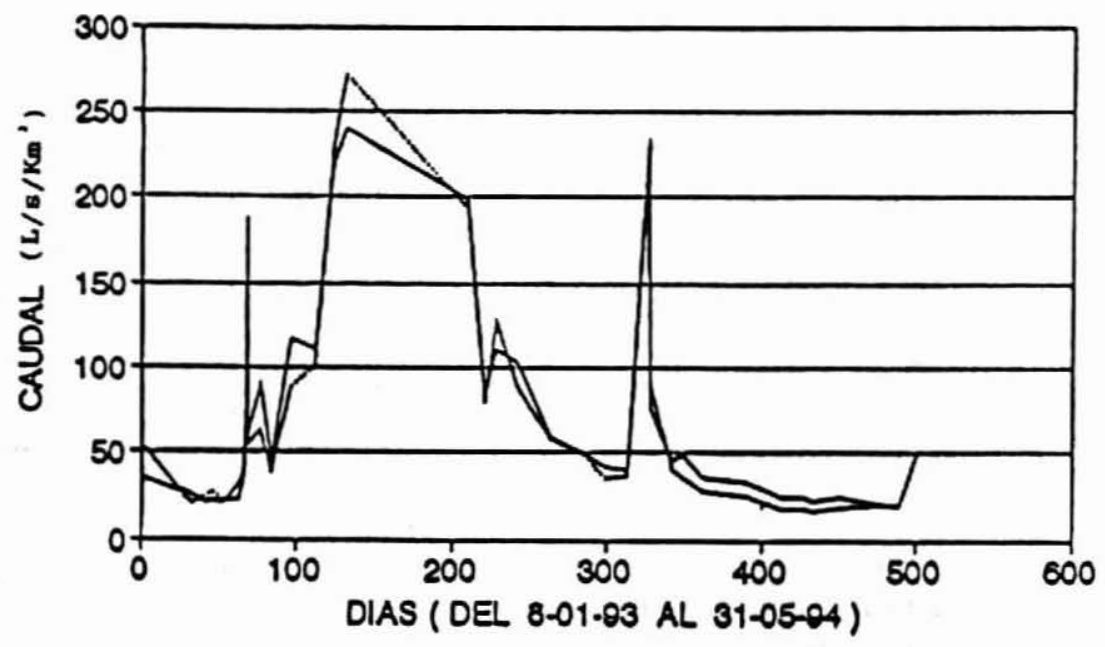

$$
\text { - PINO - NATIVO }
$$

Figura Nº 6. DISTRIBUCION DE CAUDALES PROMEDIO

A pesar de las tendencias, el análisis de varianza de todos los antecedentes de caudal (Cuadro $\mathrm{N}^{\circ}$ 2) no mostró diferencias significativas para los tratamientos Pino y Nativo con un nivel de significación de un $95 \%$ y $85 \%$, debido a que sus medias son muy similares y porque existen valores fuera de rango dentro de los dos grupos, Con los datos agrupados en mayores de 60 $\mathrm{U} / \mathrm{km}^{2}$ tampoco existieron diferencias a un nivel de $95 \%$ y $85 \%$ de significación,ya que las medias son homogéneas, Para los caudales agrupados en menores de $60 \mathrm{~L} / \mathrm{s} / \mathrm{km}^{2}$ existieron diferencias significativas al nivel de confianza de $85 \%$, presentando heterogeneidad de medias, lo que ratifica las diferencias en el sentido de un mayor rendimiento hídrico en el Nativo para las estaciones más secas del año, 


\section{Cuadro $\mathrm{N}^{\circ} 2$}

RESULTADO ANDEVA CAUDALES

\begin{tabular}{|c|c|c|c|c|c|c|}
\hline \multicolumn{7}{|c|}{ ESTADIGRAFOS } \\
\hline Variables & Cuencas & $\mathrm{N}^{\circ}$ Obervaciones & Promedio & F. Calc. & F. Tab & $\begin{array}{c}\text { Nivel } \\
\text { Significativo }\end{array}$ \\
\hline $\begin{array}{l}\text { Caudal } \\
\text { Caudal }<60 \\
\text { Caudal }>60\end{array}$ & $\begin{array}{l}\text { Pino Prom. } \\
\text { Nativo Prom. } \\
\text { Pino } \\
\text { Nativo } \\
\text { Pino } \\
\text { Nativo }\end{array}$ & $\begin{array}{l}30 \\
30 \\
18 \\
18 \\
12 \\
12\end{array}$ & $\begin{array}{r}78,4 \\
81,4 \\
33,5 \\
39,9 \\
145,7 \\
143,5 \\
\end{array}$ & $\begin{array}{l}0,029 \\
2,459 \\
0,006 \\
\end{array}$ & $\begin{array}{l}4 \\
2,35 \\
4,28 \\
\end{array}$ & $\begin{array}{l}95 \% \text { NS } \\
85 \% \text { S } \\
95 \% \text { NS }\end{array}$ \\
\hline
\end{tabular}

Del análisis de frecuencia de los caudales se desprende que la mayor cantidad de registros con caudales bajos se presenta en las cuencas Pino, encontrándose en estas el $41,37 \%$ de los registros con caudales bajo los 40 $\mathrm{Us} / \mathrm{km}^{2}$, a diferencia de las cuencas Nativo que presentó el $23,33 \%$ de los registro bajo este nivel (Figura $\mathrm{N}^{\circ} 7$ ), 


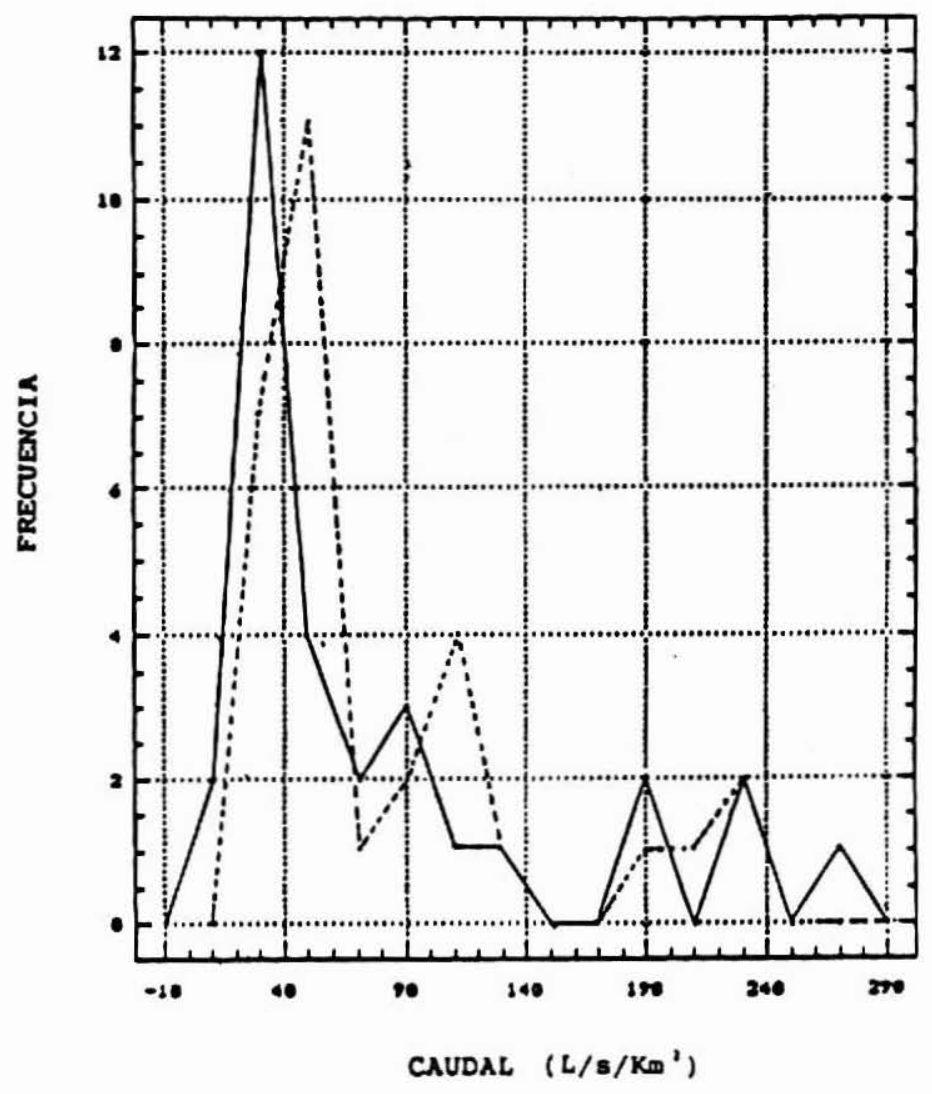

Nativo .... Pino

Figura $N^{\circ} 7$. ANALISIS DE FRECUENCIA CAUDALES

Calidad de Aguas,

La turbidez y seston $(\mathrm{mg} / \mathrm{L})$ versus caudales al momento del muestreo para las cuencas Pino y Nativo se presentan el las Figuras $N^{\circ} s 8$ y 9 , 
Para la turbidez la concentración de sedimentos en suspensión varió para las cuencas Pino entre 2,5 y $7,5 \mathrm{mg} / \mathrm{L}$ y para Nativo entre 2,5 y $6,0 \mathrm{mg} / \mathrm{L}$, En los análisis de sedimentos los niveles encontrados variaron entre 1,19 y 41,41 $\mathrm{mg} / \mathrm{L}$, para las cuencas Pino y entre 1,11 y $30,45 \mathrm{mg} / \mathrm{L}$, para las cuencas Nativo, Valores superiores se registraron en una cuenca cercana a Valdivia, que desde 0,01 a 76,13 mg/L (Rodriguez, 1991).

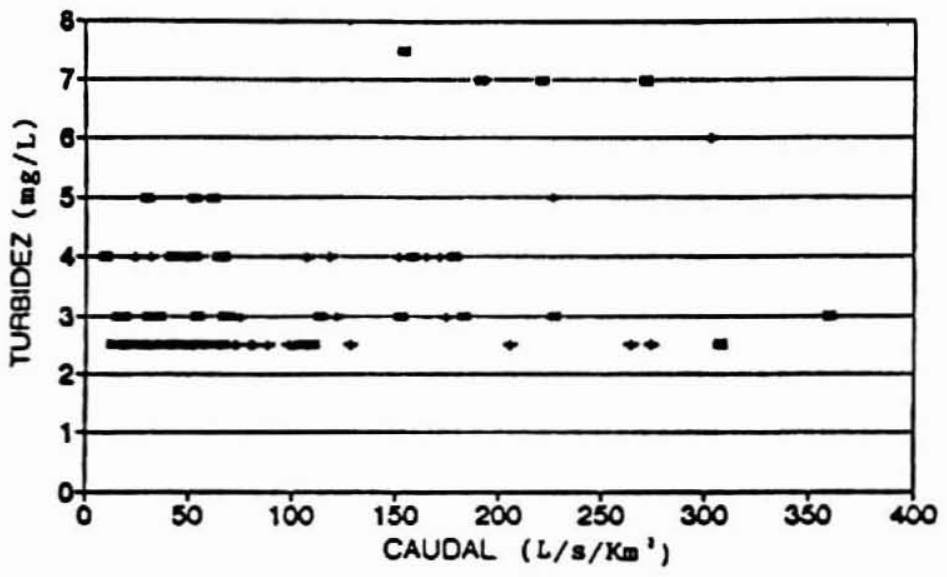

- PINO * NATIVO

Figura $N^{\circ} 8$. TURBIDEZ CUENCAS PINO Y NATIVO 


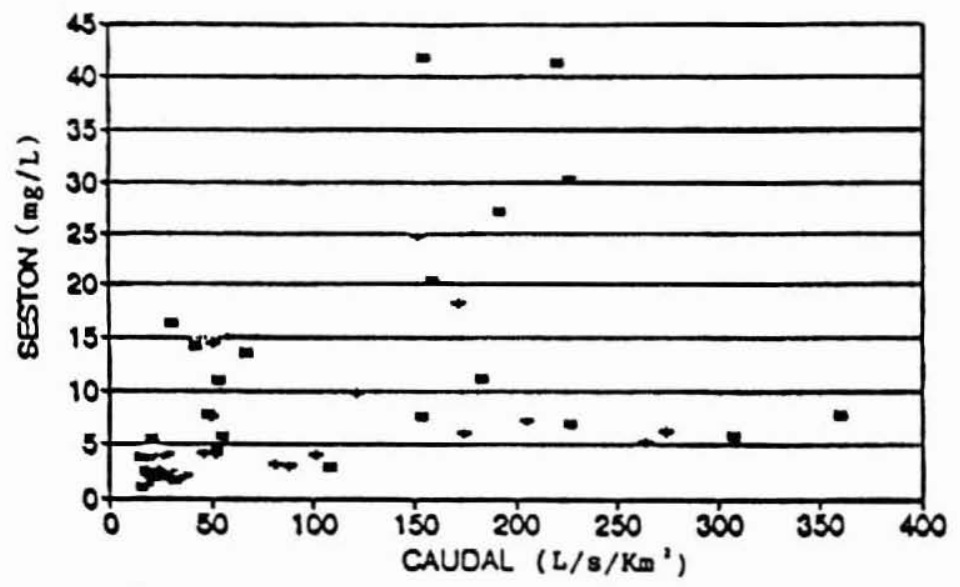

\section{- PINO * NATIVO}

Figura $\mathrm{N}^{\circ} 9$. SESTON CUENCAS PINO Y NATIVO

En ninguna de las cuencas se aprecia una relación clara entre caudal y turbidez o caudal y sedimentos, lo cual se debería a que las máximas cargas de sedimentos se producen bajo diferentes condiciones climaticas y saturación del suelo, por ejemplo lo ocurrido con las primeras lluvias de alta intensidad después de un periodo con escasas precipitaciones, Sin embargo con las lluvias intensas de invierno el efecto de arrastre puede ser comparativamente menor a pesar que los caudales son superiores, Sin embargo, se ve que las cuencas de pino generan niveles más altos tanto en turbidez como en seston,

El análisis de varianza de los antecedentes de turbidez y seston presentan diferencias significativas entre las cuencas Pino 2 y Nativo 2 y para la totalidad de los registros de Pino y Nativo (Cuadro $N^{\circ} 3$ ), 
Cuadro $N^{0} 3$

RESULTADO ANDEVA TURBIDEZ Y SESTON

\begin{tabular}{|l|l|c|c|c|c|c|}
\hline \multicolumn{9}{|c|}{ ESTADIGRAFOS } \\
\hline Variables & Cuencas & N $^{\circ}$ Obs. & Promedio & F. Calc. & F. Tab. & Nivel Significativo \\
\hline Turbidez & Pino 1 & 35 & 3,13 & & & \\
& Nativo 1 & 35 & 2,86 & 1,063 & 3,98 & $95 \%$ NS \\
& Pino 2 & 26 & 2,71 & & & $95 \% \mathrm{~S}$ \\
& Nativo 2 & 26 & 3,15 & 6,064 & 4,03 & $95 \% \mathrm{~S}$ \\
& Pino & 53 & 3,24 & & & \\
& Nativo & 55 & 2,82 & 5,426 & 3,92 & $95 \% \mathrm{NS}$ \\
\hline Seston & Pino 1 & 11 & 8,29 & & & \\
& Nativo 1 & 8 & 5,41 & 1,145 & 4,41 & \\
& Pino 2 & 10 & 9,23 & & & $95 \% \mathrm{~S}$ \\
& Nativo 2 & 9 & 4,31 & 5,559 & 4,41 & \\
& Pino & 21 & 8,74 & & & $95 \% \mathrm{~S}$ \\
& Nativo & 17 & 4,83 & 5,549 & 4,07 & \\
\hline
\end{tabular}

\section{CONCLUSIONES}

Los caudales en las cuencas cubiertas con bosques de pino no presentan diferencias significativas con los de bosque nativo para caudales instantáneos superiores a $60 \mathrm{~L} / \mathrm{s} / \mathrm{km}^{2}$, sin embargo las cuencas de pino presentan los registros de caudal instantáneo de mayor magnitud, lo que revela una tendencia a generar escorrentias de alta intensidad,

Por otra parte, las cuencas presentan diferencias significativas en el rendimiento hidrico en las estaciones del año con bajas precipitaciones (caudales inferiores a $60 \mathrm{~L} / \mathrm{s} / \mathrm{km}^{2}$ ), Las cuencas cubiertas por bosque nativo alcanzan niveles superiores en cantidad de hasta un $28 \%$ en la época de verano,

Las tasas de concentración de sedimentos en el agua de las cuencas cubiertas con plantaciones de pino son superiores a las con cubierta de bosque nativo, presentando diferencias significativas ambas cubiertas, La concentración de sedimentos en las cuencas Pino alcanza niveles de $41,4 \mathrm{mg} / \mathrm{L}$ y en las Nativos llega a $30,4 \mathrm{mg} / \mathrm{L}$, En los análisis de turbidez las diferencias 
también son significativas presentando las cuencas Pino valores máximos de $7,5 \mathrm{mg} / \mathrm{L}$ y las Nativo de $6,0 \mathrm{mg} / \mathrm{L}$,

Con los datos obtenidos no se encontró una clara relación entre caudal con turbidez o seston,

\section{REFERENCIAS BIBLIOGRAFICAS}

Alvarez, F, 1986, Técnicas y Trabajos Usados en el Manejo de Cuencas para Efectos de Producción de Agua Potable. Chile Forestal, Documento técnico (17),

Ciancaglini, N, 1987, Procedimientos de Disponibilidad de Agua, Manual de Desarrollo y Manejo de Cuencas Alto Andinas, Vol 4. Italia,

Contreras, A, En Prensa, Diagnóstico y Proposición de Pautas para el Manejo de las Microcuencas que Abastecen de Agua al Campus Isla Teja de la Universidad Austral de Chile, Tesis, Valdivia,

FAO, 1976, Some Simple Hydrological Techniques for Reconnaissance Work in Watershed Management, Forest Resources Division, FAO, Rome, 45-52,

Francke, S, 1993, Efectos de las Plantaciones Forestales en el Suelo, Chile Forestal, Documento Técnico (70),

Huber, A; Oyarzun, C; Ellies, A, 1985, Balance Hidrico en Tres Plantaciones de Pinus Radiata y una Pradera, Bosque Nº(2).

Huber, A, 1992, Redistribución de las Precipitaciones y Balance Hidrico de un Rodal de Pinus radiata y un Bosque Nativo en el Sur de Chile, en Actas de II Simposio Pinus Radiata Investigación en Chile Valdivia Chile, $6 p$.

INIA, 1989, Mapa Agroclimático de Chile, INIA, Area Agroecología, Programa Ecología y Producción. Proyecto Agrometeorología, $221 \mathrm{pg}$.

Iroume, A, 1990, Assessment of Runoff and Suspended Sediment Yield in a Partially Forested Catchment in Southern Chile, Wat, Resour. Res. . 26:2637-2642 
Iroume, A; Gayoso, J; Huber, A, 1992, Evaluación del Impacto del Plan de Manejo Forestal sobre la Cantidad y Calidad de las Aguas de las Cuencas del Estero Llancahue, ESSAL - UACH, Informe convenio $N^{\circ} 195,32 p$,

Lassen, L; Lull, H; Frank, B, 1965, Algunas Relaciones entre

Planta, Suelo y Agua en el Manejo de Cuencas, México, Agencia para el Desarrollo Internacional, 68p,

Lopez, F y Blanco, M, 1976, Hidrología Forestal, Escuela Técnica Superior de Ingenieros de Montes, Madrid, España, 60p.

Lopez, F y Pérez, A, 1981, Papel de la Vegetación en Ciclo Hidrológico, Actas de la IV Conferencia Nacional sobre Hidrología General y Aplicada, Zaragosa, España, 127142p.

Mac Donald Lee H, 1991, Monitoring. Guidelines to Evaluate Effects of Forestry Activities on Stream in the Pacific Northwest and Alaska, United States Enviromental Protection Agency, Washintong 166p.

Otero L, Donoso H, Barrales L, 1993, Análisis de los Efectos Ambientales del Reemplazo de Bosque Nativo por Plantaciones, Instituto Forestal, Informe Interno, Unidad de Medio Ambiente,

Rodríguez, D, 1991, Escorrentía y Producción de Sedimentos en Suspensión en una Cuenca de la Décima Región, Tesis Universidad Austral de Chile, Valdivia 38p, 